\title{
ON THE SOLUTION OF EXTERIOR PLANE PROBLEMS BY THE BOUNDARY ELEMENT METHOD: A PHYSICAL POINT OF VIEW
}

\author{
G. Bonnet ${ }^{\mathrm{a}}$, A. Corfdir ${ }^{\mathrm{b}}$, M.T. Nguyen ${ }^{\mathrm{a}, \mathrm{c}}$ \\ ${ }^{a}$ Université Paris Est, Laboratoire Modélisation et Simulation Multi-Echelle, MSME UMR \\ 8208 CNRS, 5 boulevard Descartes, 77454 Marne la Vallée Cedex, France \\ ${ }^{b}$ Université Paris-Est, Laboratoire Navier, UMR 8205 CNRS - IFSTTAR - ENPC, Ecole \\ des Ponts ParisTech, 6-8 av. Blaise Pascal, 77455 Marne-la-Vallée, France \\ ${ }^{c}$ Institute of Mechanics, 264 Doican, Hanoi, Vietnam
}

\begin{abstract}
The paper is devoted to the solution of Laplace equation by the boundary element method. The coupling between a finite element solution inside a bounded domain and a boundary integral formulation for an exterior infinite domain can be performed by producing a "stiffness" or "impedance matrix" which is equivalent to the interaction coming from BEM, when coupled with FEM stiffness or impedance matrix. It is shown in a first step that the use of classical Green's functions for plane domains can lead to impedance matrices which have eigenvalues of different signs, which is physically and numerically unsatisfying and also to singular impedance matrices, corresponding to the classical degenerate scale problem. Avoiding the degenerate scale problem is classically overcome by adding to the Green's function a constant which is large compared to the size of the 2D domain. However, it is shown that this constant affects the solution of exterior problems in the case of non-null resultant of the normal gradient at the finite boundary. It becomes therefore important to precise the value of this constant related to a characteristic length introduced into the Green's function. Using a "slender body theory" allows to show that for long cylindrical domains with a given cross section, the characteristic length is asymptotically equal to the length of the cylindrical domain.Comparing numerical or analytical $3 \mathrm{D}$ and 2D solutions on circular cylindrical domains confirms this result for circular cylinders.
\end{abstract}

Keywords: Elasticity, Laplace equation, Boundary Element Method, Plane problems, Green's function, Exterior problem, Impedance matrix

\section{Introduction}

The advantage of the boundary element method, compared to other approximate solutions is the most obvious when the method is used for problems on 
unbounded domains, either for solving completely linear systems of partial differential equations or very often as a complement of the finite element method in the case of problems containing local non-linearities.

It is well known that, in the case of $2 \mathrm{D}$ problems, the fundamental solution tends to infinity when the distance between source and observation point tends to infinity, either for the case of Laplace equation or for the elasticity operator. A consequence is that if the resultant of sources is not null, the solution does not vanish at infinity. A main point of interest in the literature is the wellposedness of the Exterior Boundary Value Problem. Due to the logarithmic behaviour of the fundamental solution at infinity, the well-posedness has been studied primarily in the case where the resultant of sources (or forces in problems related to elasticity equations) on the finite boundary is null $[1,2,3,4,5,6,7]$. However many practical problems need the application of sources or forces whose resultant is non-null; this case has been considered in $[8,9]$. The validness of the corresponding integral equation has been proved in details by [10] for the standard integral equation using Somigliana equality (with a new kernel) and by [11] for the regularized integral equation without restriction on the kernel which is used. So, in this paper, we shall assume that the consideration of an exterior problem by standard BEM is licit even if the resultant of sources or forces applied at the boundary is non-null.

A second difficulty related to $2 \mathrm{D}$ problems is the loss of uniqueness of the solution when the domain under consideration has specific dimensions and when the classical logarithmic function for an infinite domain is used. It was early recognized [12] and the usual practical way to circumvent this problem is to add a constant to the Green's function which must be adjusted to the dimensions of the domain $[13,9]$. This method leads however to the obtaining of the potential up to an additional arbitrary constant value, this method being related to a convenient "scaling" of the distances introduced when using the fundamental solution [14]. Other methods are used to perform a "regularization" of the problem and to recover the uniqueness $[15,16,17,18,19,20]$. This problem is recurrent within the literature $[21,22,7,23,24]$. The link between the condition number of the BEM matrices and the scaling of the problem has been also investigated [25]. Two points remain unclear in this context. First, it is clear that some constants added to the fundamental solution must be avoided, leading to non-uniqueness of the solution. However, it is not always clear if all other values can still be used. Indeed, it is practically always considered that the constant is arbitrary and that all solutions related to different values of the arbitrary constant are the same, upto an additional constant. This paper addresses these two points in the case of Laplace equation. The purpose of the present paper is therefore :

- to present in section 2 an example where the use of the classical singular solution for Laplace equation within the usual formulation of the boundary integral equation leads to non physical solutions. The example recovers the loss of uniqueness for some specific problems and shows that in addition to the loss of uniqueness, some fundamental solutions can lead to a loss of positiveness of the "impedance matrix" induced by the boundary element formulation. 
- to prove next that for interior problems any constant added to the fundamental solution does not change the solution which is given up to an added constant and that in the case of exterior problems, solutions related to different constants added to the fundamental solution are different for Dirichlet or mixed value problems. Therefore, it is clearly of importance to find the value of the "right" constant related to a given physical problem or the right "characteristic length" associated to this problem.

- to show in section 3 by using the "slender body theory" that the solution to a given $2 \mathrm{D}$ boundary value problem can be obtained as the asymptotic limit of the solution to an associate 3D problem over a long cylinder as soon as the associated "characteristic length" is equal to the length of the cylinder.

- to confirm in section 6 the result of the "slender body theory" by comparing the results coming from 3D problems over long cylinders, these results being obtained by various analytical (section 4) or numerical (section 5) solutions.

\section{Discussion of the direct formulation of the boundary element method in the case of the exterior problem for Laplace equation}

2.1. Example of loss of uniqueness and appearance of unphysical results on a numerical solution built from the classical formulation of the exterior problem

Our discussion will start with the classical formulation of the exterior "Dirichlet" Boundary Value Problem. Let us therefore consider the solution $u$ of the exterior problem of Laplace equation on a plane domain $D$ having a boundary $\partial D$ for Dirichlet boundary conditions. The classical discretized integral equation writes (see appendix A):

$$
[H][u]=[G][q]
$$

where $[u]$ and $[q]$ contain the nodal displacements and the nodal values of the normal flux while $[H]$ and $[G]$ are built from the interpolation functions, the geometry of the elements and from the Green's function (and its derivatives).

The Green's function which is used at the beginning is the classical expression for $2 \mathrm{D}$ problems given by:

$$
G=\frac{1}{2 \pi} \ln (1 / r)
$$

$\mathrm{r}$ being the distance between source and observation point.

The physical soundness of the results will be studied by using the eigenvalues of:

- the matrix $[G]$,

- the symmetric part $[K]$ of the "impedance matrix" $\left[K_{1}\right]$ (cf appendix A) which allows the computation of the supply of energy from:

$$
W=\frac{1}{2}[u]^{T}[F]=\frac{1}{2}[u]^{T}\left[K_{1}\right][u]=\frac{1}{2}[u]^{T}[K][u]
$$




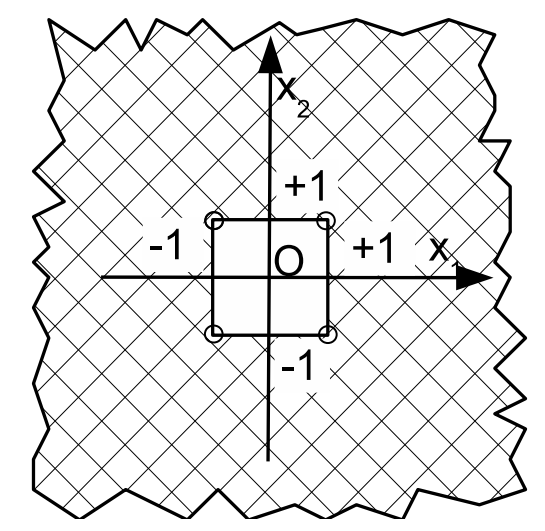

Figure 1: The exterior 2D problem outside a square.

where $\left[K_{1}\right]$ is the "impedance matrix" which is built by using matrices $[H]$ and $[G]$ as in $[26]$ :

$$
\left[K_{1}\right]=[Q][G]^{-1}[H]
$$

where $[Q]$ is a matrix allowing the condensation of boundary stresses on nodal forces.

It may be noticed that the "impedance matrix" $\left[K_{1}\right]$ is in general not symmetric, due to the fact that $[G]$ and $[H]$ are generally themselves not symmetric. Let us consider a simple example which is the problem exterior to a square having a side length equal to 2, as shown in Fig.1.

\begin{tabular}{|l|l|l|l|}
\hline \multicolumn{3}{|c|}{ Eigenvalues of $[\mathrm{K}]$} \\
\hline-6.06 & 1.58 & 1.58 & 1.72 \\
\hline
\end{tabular}

Table 1: Eigenvalues of the matrix $[K]$ when using the classical $2 D$ Green's function for Laplace equation in the domain of Fig.1.

Table 1 displays the eigenvalues of matrix $[K]$, showing that one of these eigenvalues is negative, which is physically inconsistent, because it implies a negative supply of energy for some boundary conditions. Now, let us show that this situation is closely related to the well-known problem of loss of uniqueness of the Dirichlet problem which is mentioned in many papers. Indeed, Fig.2 displays the smallest eigenvalue of matrix $[G]$ for different sizes $L$ of the side of the square defining the boundary of the exterior problem, noticing that for any value of $L$, all other three eigenvalues of $[G]$ are positive.

Fig.2 shows that the smallest eigenvalue of matrix $[G]$ vanishes when $L=$ $L_{\max }=1.543$, becomes positive when $L<L_{\max }$ and is negative when $L>$ 
$L_{\max }$. It is worthwhile noticing that the critical length $L_{\max }$ is not far from the value obtained by [25] from different considerations, despite the roughness of the boundary discretization in our example. The value $L=L_{\max }$ corresponds

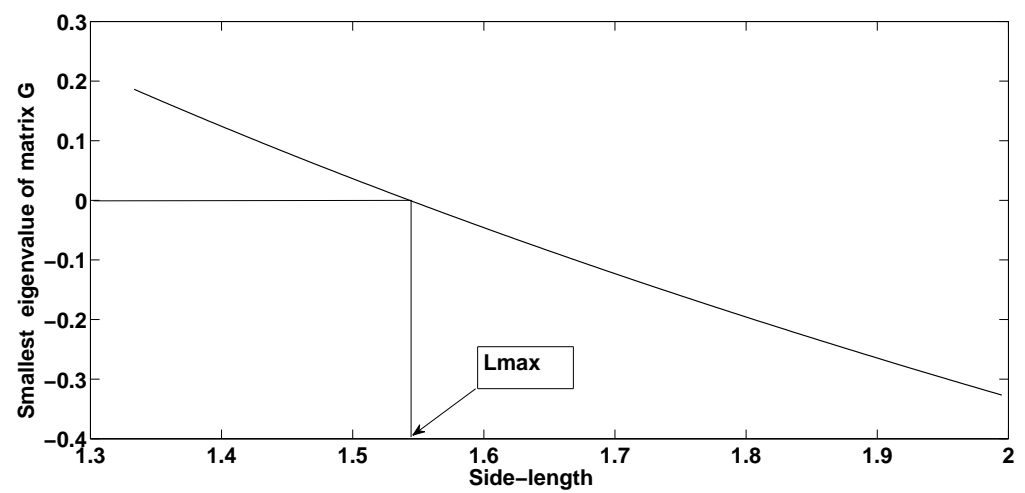

Figure 2: Smallest eigenvalue of matrix $[G]$ for different side-lengths.

to a matrix $[G]$ which is singular and therefore to the loss of uniqueness of the discretized Dirichlet boundary value problem. Let us now look at the extreme

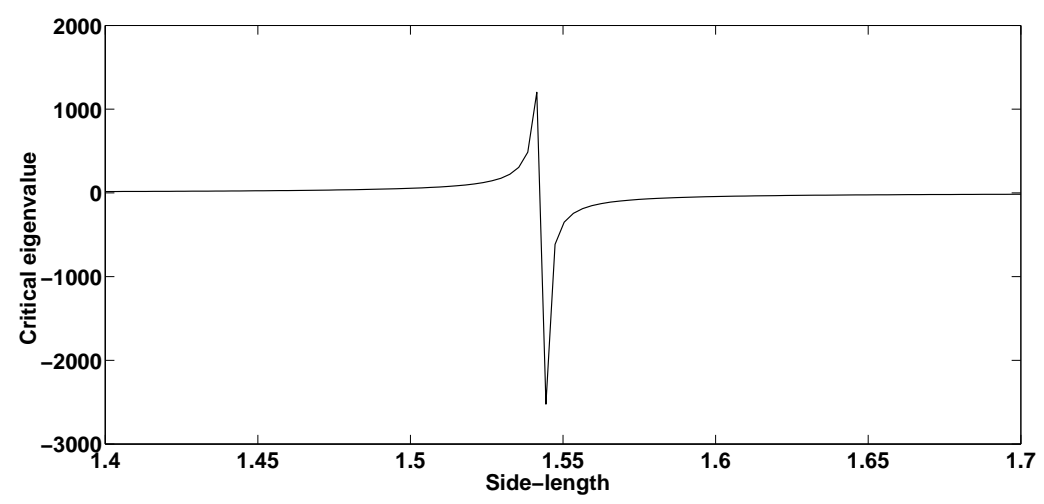

Figure 3: Critical eigenvalue of matrix $[K]$ for different side-lengths.

eigenvalues of matrix $[K]$. Fig.3 displays the smallest eigenvalue of matrix $[K]$ as a function of $L$, when it is negative and the largest eigenvalue of this matrix when all eigenvalues are positive, showing that the smallest eigenvalue of $[K]$ is also negative for all values of $L>L_{\max }$. However, the negative eigenvalue tends to $-\infty$ when $L$ comes near $L_{\max }$ with $L>L_{\max }$, coming obviously from the fact that $[K]$ is built from $[G]^{-1}$. Obviously, the largest eigenvalue of $[K]$ tends to infinity when $L$ comes near $L_{\max }$ with $L<L_{\max }$, meaning that even if $[K]$ has all its eigenvalues positive, the supply of energy can be unphysically large when $L$ comes near $L_{\max }$. These results recover from a different point of view 
the results obtained in (Dijkstra and Mattheij, 2007) on the relation between the logarithmic capacity of interaction matrices and the condition number of $[G]$ matrix.

So, this simple example shows that the boundary value problem related to Dirichlet boundary condition may not be sound from a physical point of view. It may lead either to non-uniqueness or to an unphysical supply of energy, depending of the scale of the problem. There are two equivalent means to overcome the problem: either effect a "scaling", on the physical lengths defining the boundary of the problem, or add a constant to the Green's function of the problem. These solutions are equivalent. Indeed, this is generally achieved by using a Green's function with the form:

$$
G=\frac{1}{2 \pi} \ln (l / r)=\frac{1}{2 \pi} \ln (1 / r)+C
$$

with $C=\frac{1}{2 \pi} \ln (l)$.

So, the main question is the choice of $C$ or $l$. As shown before, these constants must be chosen in a convenient range of values to ensure uniqueness and positiveness of the impedance matrix. A current view is to consider that all solutions are the same upto an additional constant. This is the question which will be studied in the following.

\subsection{Comparison of solutions related to different constants added to the funda- mental solution}

\subsubsection{Interior problem}

We consider the regularized integral equation for Laplace equation for interior problems and for fundamental solutions which are equal up to a constant $C$, given by:

$$
\int_{\partial D}\left(u(\mathbf{y})-u(\mathbf{x}) \frac{\partial G(\mathbf{x}, \mathbf{y})}{\partial \mathbf{n}(\mathbf{y})} \mathrm{d} S_{y}=\int_{\partial D} \frac{\partial u}{\partial \mathbf{n}}(G(\mathbf{x}, \mathbf{y})+C) \mathrm{d} S_{y}\right.
$$

Without internal sources, the integral $\int_{\partial D} \frac{\partial u}{\partial \mathbf{n}} \mathrm{d} S_{y}$ is null, due to energy conservation. So, the solution of the integral equation does not depend of the constant $C$. Therefore, the solution is perfectly defined as soon as Dirichlet boundary condition is applied at least at one point and does not depend on the constant introduced into the Green's function. The constant added to the Green's function appears in the conditioning of the digitized system of equations, but not in the solution. For purely Neumann's boundary conditions, the solution is defined upto an arbitrary constant, contrarily to the case of the exterior problem, as shown thereafter.

\subsubsection{Exterior problem}

For the exterior problems, the regularized integral equation reads:

$$
u(\mathbf{x})+\int_{\partial D}\left(u(\mathbf{y})-u(\mathbf{x}) \frac{\partial G(\mathbf{x}, \mathbf{y})}{\partial \mathbf{n}(\mathbf{y})} \mathrm{d} S_{y}=\int_{\partial D} \frac{\partial u}{\partial \mathbf{n}}(G(\mathbf{x}, \mathbf{y})+C) \mathrm{d} S_{y}\right.
$$


As explained in the introduction, the boundary conditions for an exterior problem do not need to be at equilibrium on the finite boundary. Therefore, the integral $\int_{\partial D} \frac{\partial u}{\partial \mathbf{n}} \mathrm{d} S_{y}$ is not null. As a consequence, the part containing the constant term does not vanish and the solution depends explicitly of this constant term.

At this stage, it can be seen that the problem of the constant which is added to the Green's function is not crucial in the case of the interior problem as soon as the uniqueness is ensured, because the numerical solution does not depend on the value of the constant added to the Green's function. In the case of the exterior problem, the solution of the boundary value problem by the boundary element method depends explicitly of the constant added to the Green's function. In the case of Neumann's boundary conditions, all solutions related to different constants added to the Green's function are equal upto a constant. Indeed, let us now consider two solutions which are equal, up to a constant $C_{u}$. It can be seen from equation(7) that $C_{u}$ is related to the constant $C$ by:

$$
C_{u}=C \cdot \int_{\partial D} \frac{\partial u}{\partial \mathbf{n}} \mathrm{d} S_{y}
$$

So, if one considers the solutions for Neumann's boundary conditions related to two values which are different by the constant $C$, they are equal upto an added constant $C_{u}$ given by this relation, which involves the resultant of the flux through the finite boundary. As for the interior problem, the constant added to the Green's function does not modify the solution if the resultant of the flux at the finite boundary is null.

In the case of mixed boundary conditions, the solutions corresponding to different values of the constant added to the Green's function can have very different forms. So, it becomes crucial to define precisely the value of the constant added to the Green's function. This is the main question addressed in this paper.

The consequence of the choice of the constant in the Green's function on the positiveness of the impedance can be seen in the example of an exterior problem with a circular boundary on which a constant potential is imposed. The classical solution of the problem is

$$
u=A \cdot \ln (r)+B
$$

where $A$ and $B$ depend of the boundary conditions.

The boundary impedance related to the solution of this problem can be recovered from the boundary integral formulation

Indeed, let us consider the exterior problem related to a boundary condition $u=u_{0}$ at $r=R$. The integral equation becomes:

$$
u(\mathbf{x})+\int_{\partial D}(u(\mathbf{y})-u(\mathbf{x})) \frac{\partial G(\mathbf{x}, \mathbf{y})}{\partial \mathbf{n}(\mathbf{y})} \mathrm{d} S_{y}=\int_{\partial D} \frac{\partial u}{\partial \mathbf{n}}(\mathbf{y}) G(\mathbf{x}, \mathbf{y}) \mathrm{d} S_{y}
$$

For a constant potential at the boundary, the second integral vanishes and the normal gradient $\frac{\partial u}{\partial \mathbf{n}}=q$ is constant. Then the integral equation produces: 


$$
u_{0}=q_{0} \int_{\partial D} \frac{1}{2 \pi} \ln (l / r) \mathrm{d} S_{y}
$$

with $r=\|\mathbf{y}-\mathbf{x}\|$.

The integration leads finally to:

$$
u_{0}=q_{0} R \ln (l / R)
$$

which shows that the impedance $\frac{q_{0}}{u_{0}}$ at the boundary depends of the characteristic length $l$ and can be negative for small values of $l$.

\subsection{Obtaining a physically satisfying solution}

If one wants to recover solutions which are physically satisfying, it is necessary to use a characteristic length which has a physical meaning, for the problem under solution. This problem is obviously closely related to the non physical nature of 2D solutions within an infinite plane which do not vanish at infinity.

From another point of view, 2D problems are usually encountered in two cases:

- plane problems on domains which are very thin along one direction (typically plates or plane stress elasticity). These problems are only of the interior kind.

- problems whose solutions correspond to fields which do not depend on one space variable (typically plane strain elasticity). These problems can be either of interior or exterior kind.

In this last case, the field is generally an approximation in a given restricted domain of a 3D field solution of a 3D problem defined on all space.

So, it is natural in this last case to associate to the 2D problem a $3 \mathrm{D}$ problem on a long cylinder, as in Fig.4, this cylinder having not necessarily a circular section. The conditions on the lateral boundary of the cylinder are deduced from the boundary conditions on the boundary of section $\Gamma$. The cylinder can be either closed at its endings and in this case boundary conditions must be added on the related surfaces $B_{\alpha}, B_{-\alpha}$. In the following, it will be shown that the restriction of the solution of the "associated 3D problem" to the central section of the cylinder is approximated by the solution of the $2 \mathrm{D}$ problem in the vicinity of the cylinder as soon as the length of the cylinder is long enough.

This question will be studied by using three different approaches. In section 3 , the comparison of the 2D and 3D solutions will be effected by using a method usually known as the "slender body theory" which consists in matching 2D solution and 3D solution far from the cylinder in the midplane of the cylinder. This solution will be provided for Neumann boundary solutions along the surface of the cylinder. Next, in the following sections, the simplified case of a circular cylinder will be studied. In section 4, known analytical solutions will be used for obtaining the explicit impedance in the midplane obtained for Dirichlet boundary conditions corresponding to a given constant potential at the cylinder. Finally in section 5 , the problem related to a circular cylinder will be solved by using boundary elements for Dirichlet and Neumann boundary conditions. 


\section{Relation between $2 \mathrm{D}$ and $3 \mathrm{D}$ problem obtained by the slender body theory}

\subsection{Characteristic length obtained from the slender body theory}

The method proposed in the following is to recover the same kind of potential as in the previous subsection by starting from the well-posed 3D problem. We will use a method based on the matching of a $2 \mathrm{D}$ solution and a $3 \mathrm{D}$ solution in some area of the plane $x_{3}=0$. Similar methods based on similar principles are used in fluid mechanics [27, 28] and also in solid mechanics [29, 30].

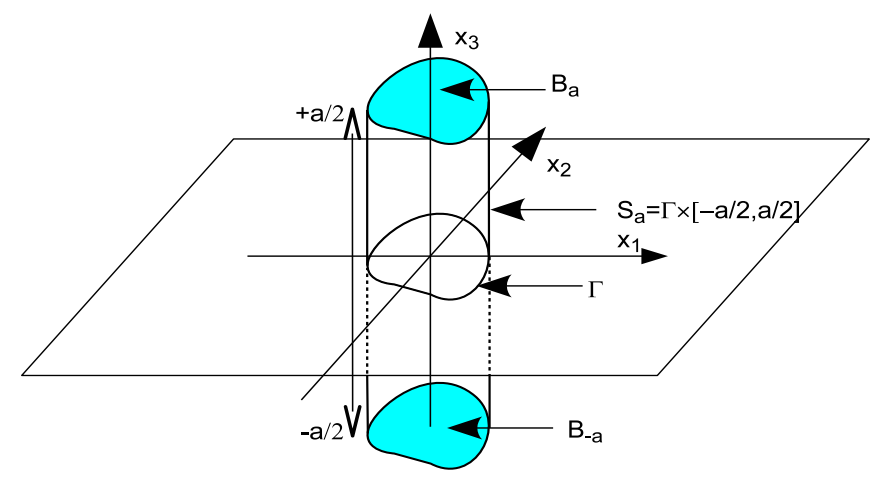

Figure 4: The exterior 2D problem viewed as a part of the limit of a $3 D$ problem.

A 2D Neumann exterior problem on the domain bounded by $\Gamma$ with boundary condition $\partial u\left(x_{1}, x_{2}\right) / \partial \mathbf{n}=f\left(x_{1}, x_{2}\right)$ on $\Gamma$ is considered as the limit when $a$ tends to infinity of a 3D Neumann problem (Figure 4) with the following boundary condition on its boundary $\partial D_{a}$ : on $S_{a}, \partial u_{a}\left(x_{1}, x_{2}, x_{3}\right) / \partial \mathbf{n}=f\left(x_{1}, x_{2}\right)$, on $B_{a} \cup B_{-a}, \partial u_{a}\left(x_{1}, x_{2}, x_{3}\right) / \partial \mathbf{n}=0$, with $\partial D_{a}=S_{a} \cup B_{a} \cup B_{a}$. The "diameter" of $\Gamma$ is defined by $d=\max \sqrt{\left(x_{1}-y_{1}\right)^{2}+\left(x_{2}-y_{2}\right)^{2}},(\mathbf{x}, \mathbf{y}) \in \Gamma$.

We first consider the $3 \mathrm{D}$ problem. The integral representation of solution $u_{a}$ is:

$$
u_{a}(\mathbf{x})=\int_{\partial D_{a}} \frac{\partial u_{a}}{\partial \mathbf{n}}(\mathbf{y}) G_{3 D}(\mathbf{x}, \mathbf{y}) \mathrm{d} S_{y}-\int_{\partial D_{a}} u_{a}(\mathbf{y}) H_{3 D}(\mathbf{x}, \mathbf{y}) \mathrm{d} S_{y}
$$

We assume here that $\int_{\partial D_{a}} \frac{\partial u_{a}}{\partial \mathbf{n}}(\mathbf{y}) \mathrm{d} S_{y} \neq 0$, otherwise the solution does not depend on the choice of the constant in the Green's function (see 2.2.2). The dominant term in the last equation is the first integral when $\mathrm{r}$ tends to infinity, but some care is needed because the second term depends on $a$ and is bounded by $A$.sup $\left|u_{a}\right|(d / r), A$ being a constant depending on $\Gamma$. We will assume that $\sup _{\partial D_{a}}\left|u_{a}\right|=\mathrm{O}(\ln (a / d))$. $\partial D_{a}$

In fact, this conjecture is suggested by the results of numerical computations in the case of axisymmetry which are shown thereafter in section 6.2 . The figure 
9 shows that for the Neuman problem considered, the maximum value of $u$ on the boundary is obtained at $z=0$. The figure 8 shows that the $3 \mathrm{D}$ solution for $z=0$ converges to the $2 \mathrm{D}$ solution when $a \rightarrow \infty$. As this solution is given by equation (12) with $l=a$, we can conclude that $\sup _{\partial D_{a}}\left|u_{a}\right|=\mathrm{O}(\ln (a / d))$ in this case.

If $a \gg r=\sqrt{x_{1}^{2}+x_{2}^{2}} \gg d$, we have the following asymptotic value of (13) where $F=\int_{\Gamma} f(\mathbf{y}) \mathrm{d} S_{y}$ :

$$
\begin{aligned}
& u_{a}\left(x_{1}, x_{2}, x_{3}=0\right)= \\
& =\frac{1}{4 \pi} \int_{\Gamma} \int_{-a / 2}^{a / 2} \frac{\partial u_{a}}{\partial \mathbf{n}} G_{3 D}(\mathbf{x}, \mathbf{y}) \mathrm{d} y_{3}+\frac{d}{r} \mathrm{O}\left(\ln \left(\frac{a}{d}\right)\right) \\
& =\frac{1}{4 \pi} \int_{\Gamma} \frac{\partial u_{a}}{\partial \mathbf{n}} \ln \left(\frac{a / 2+\sqrt{(a / 2)^{2}+\left(x_{1}-y_{1}\right)^{2}+\left(x_{2}-y_{2}\right)^{2}}}{-a / 2+\sqrt{(a / 2)^{2}+\left(x_{1}-y_{1}\right)^{2}+\left(x_{2}-y_{2}\right)^{2}}}\right)+\frac{d}{r} \mathrm{O}\left(\ln \left(\frac{a}{d}\right)\right) \\
& =\frac{1}{4 \pi} \int_{\Gamma} f \ln \left(\frac{\left(a / 2+\sqrt{(a / 2)^{2}+\left(x_{1}-y_{1}\right)^{2}+\left(x_{2}-y_{2}\right)^{2}}\right)^{2}}{r^{2}}\right)+\frac{d}{r} \mathrm{O}\left(\ln \left(\frac{a}{d}\right)\right) \\
& =\frac{F}{2 \pi} \ln \left(\frac{a}{r}\right)+\mathrm{O}\left(\frac{r^{2}}{a^{2}}\right)+\frac{d}{r} \mathrm{O}\left(\ln \left(\frac{a}{d}\right)\right)
\end{aligned}
$$

If we consider the solution $u_{a}$ in the domain such that $r / d>\left(\ln \left(\frac{a}{d}\right)\right)^{2}$ then:

$$
u_{a}\left(x_{1}, x_{2}, x_{3}=0\right)=\frac{F}{2 \pi} \ln \left(\frac{a}{r}\right)+\mathrm{O}\left(\frac{r^{2}}{a^{2}}\right)+\mathrm{O}\left(\left(\frac{d}{r}\right)^{\frac{1}{2}}\right)
$$

We assume that the 3D problem in the midplane can be approximated by a plane problem. The integral representation for the plane problem is:

$$
u\left(x_{1}, x_{2}\right)=\int_{\Gamma} \frac{\partial u}{\partial \mathbf{n}}(y) G_{2 D}(\mathbf{x}, \mathbf{y}) \mathrm{d} S_{y}-\int_{\Gamma} u(\mathbf{y}) H_{2 D}(\mathbf{x}, \mathbf{y}) \mathrm{d} S_{y}
$$

We set: $G_{2 D}(\mathbf{x}, \mathbf{y})=1 / 2 \pi \ln (b / r) ; b$ is the arbitrary constant which appears when solving $2 \mathrm{D}$ problems. If $r \gg d$, we have the following asymptotic value:

$$
u(\mathbf{x})=\frac{F}{2 \pi} \ln \left(\frac{b}{r}\right)+\mathrm{O}\left(\frac{d}{r}\right)
$$

From comparing the 2D (17) and 3D (14) asymptotic values, one deduces the relevant choice for the constant $b$ is $b=a$. This result is compatible with our assumption on $\sup _{\partial D_{a}}\left|u_{a}\right|$. To approximate the 3D problem (with Neumann boundary condition) related to a domain which is very long in the direction of $x_{3}=0$ by a $2 \mathrm{D}$ problem, it is nessary to adopt the Green's function with $a$ the length of the domain:

$$
G_{p}=\frac{1}{2 \pi} \ln \left(\frac{a}{r}\right)
$$


i.e to fix the characteristic length used for overcoming non-uniqueness problems at the value $l=a$.

Such a Green's function with a physical definition of the related characteristic length will be called "physical Green's function" in the following. It is shown below that this Green's function corresponds to the asymptotic field produced by a line source having length $a$.

\subsection{Construction of the physical Green's function from a long line source}

It is shown now that this 2D Green's function corresponds exactly to the asymptotic value of the field produced by a constant line density located along the generating line of the cylinder related to the point source of the $2 \mathrm{D}$ boundary. Indeed, let us consider the potential produced by a finite line source parallel to the axis $O y_{3}$, centered at a point in the plane $O y_{1} y_{2}$ and having a length $a$.

The potential produced at a point $\left(y_{1}, y_{2}, 0\right)$ by that line source cutting the plane $\left(O y_{1} y_{2}\right)$ at the position $\left(x_{1}, x_{2}, 0\right)$ is given by an easy integration, leading to:

$$
u_{a}=\frac{1}{4 \pi} \ln \left(\frac{a+\sqrt{4 \cdot r^{2}+a^{2}}}{-a+\sqrt{4 r^{2}+a^{2}}}\right)
$$

where $r=\sqrt{\left(y_{1}-x_{1}\right)^{2}+\left(y_{2}-x_{2}\right)^{2}}$.

The asymptotic value when $a$ is large is then given by:

$$
u_{a}=\frac{1}{2 \pi} \ln \left(\frac{a}{r}\right)+\mathrm{O}\left(r^{2} / a^{2}\right)
$$

which is exactly, at the first order, the singular solution obtained from the slender body theory.

From another point of view, it introduces a natural characteristic length related to the dimension of the domain under study in the direction perpendicular to the considered plane, as explained in the previous section.

The result obtained so far corresponds to the asymptotic value for long cylinders. However, it is obviously of interest to verify this result by solutions related to finite cylinders in order to see at which values of large cylinder lengths, the asymptotic solution becomes satisfying. This will be the topic of the following sections.

\subsection{Discussion in relation with non-uniqueness}

As stressed before, the constant added to the Green's function must be large enough to ensure the uniqueness of Dirichlet's problem. It means that the characteristic length $l$ in the Green's function must also be large enough. More precisely, it was shown in [31] that the condition $l>R_{c}$ is sufficient, where $R_{c}$ is the radius of the circle which is circumscribed to the $2 \mathrm{D}$ boundary. It can be seen that this condition is verified by the physical Green's function corresponding to $l=a$ in the case $a \gg R_{c}$. 


\section{Analytical solutions of the Dirichlet problem for long bodies and cylinders}

This problem is widely documented in the literature for a constant value of the potential, because it corresponds to the field produced by a cylindrical conductor which is submitted to a given charge. The solution of this problem produces the capacity of the conductor, which is in practice very useful. This problem has been studied by using two different kinds of methods. The first one corresponds to the solution for a thin straight wire and is produced again by an asymptotic analysis. The second one corresponds to the analytical solution of the field produced by a finite circular cylinder.

\subsection{Asymptotic analysis for a thin straight wire}

This analysis is an old problem which was first studied by Maxwell [32] and revisited in [33]. The question is : for a given long conductor, what is the distribution of the charge linear density $\lambda$ as a function of the coordinate $z$ along the length of the cylinder? A first remark is that for a long ellipsoidal conductor, the linear charge density $\lambda$ is constant and independent of the ratio of minor to major axis. Next, Jackson [33] shows that for a long conductor with a variable radius $R(z)$ such that $R^{2}(z)=\rho^{2} . f(z)$ and a length $a=2 \alpha$ the solution is governed by its asymptotic behaviour when $\rho / \alpha \ll 1$, which is expressed by using the variable $\Lambda=2 \ln (a / \rho)$ with the form:

$$
\lambda(\zeta)=\lambda_{0} 1-\frac{1}{\Lambda} \ln \left(\frac{1-\zeta^{2}}{f(\zeta)}\right)+\frac{1}{\Lambda^{2}}\left[\left[\ln \left(\frac{1-\zeta^{2}}{f(\zeta)}\right)\right]^{2}-I\right]
$$

where $\zeta=z / \alpha$ and $I$ can be found when the shape function $f(z)$ is known.

This linear charge density distribution ensures that the potential $u$ is constant along the wire with an approximation level corresponding to $\frac{1}{\Lambda^{2}}$, its leading term being given by:

$$
u=\frac{\lambda_{0} \Lambda}{4 \pi}
$$

which is exactly the value given by (11) at the surface of the conductor by the $2 \mathrm{D}$ solution.

Indeed, due to the Gauss theorem, the normal gradient is related to the linear charge density in the median plane by:

$$
\lambda=2 \pi \cdot R(0) \cdot \frac{\partial u}{\partial \mathbf{n}}=2 \pi \cdot R(0) \cdot q(0)
$$

Combining these two last equations provides the relation between the potential and the value of the normal gradient in the midplane

$$
u=R(0) \cdot \ln (a / R(0)) \cdot q(0)
$$

which is the same as equation (12) with $a=l$. 
It is noteworthy that the second term in equation (21) provides the well known weak singularity at the tips of the long conductor. However, this singularity does not affect the charge density in the neighbourhood of the median plane, at the first order, as shown by the relation providing $\lambda(\zeta)$.

For a cylindrical conductor with circular cross-section, the constant $I$ can be exactly computed, which provides, up to second order in $\frac{1}{\Lambda}$ :

$$
\lambda(\zeta)=\lambda_{0} 1-\frac{1}{\Lambda} \ln \left(1-\zeta^{2}\right)+\frac{1}{\Lambda^{2}}\left[\left[\ln \left(1-\zeta^{2}\right)\right]^{2}+\frac{1}{2}\left[\ln \left(\frac{1+\zeta}{1-\zeta}\right)\right]^{2}-\frac{\pi^{2}}{6}\right]
$$

This leads in the mean plane to the linear charge density:

$$
\lambda=\lambda_{0}\left(1-\frac{\pi^{2}}{6 \Lambda^{2}}\right)
$$

This relation allows to obtain the correction at the second order in $1 / \Lambda$ between the impedance produced in the median plane by both $2 \mathrm{D}$ and $3 \mathrm{D}$ solutions. Indeed, the potential is now given by:

$$
u=\frac{1}{1-\frac{\pi^{2}}{6 \Lambda^{2}}} R \cdot \ln (a / R) \cdot q(0)
$$

It must be noticed that the convergence is slow, because $\Lambda$ involves a logarithmic dependence as a function of the aspect ratio $a / R$. However, the convergence is better for a cylinder than for the most general long body, because the difference between $2 \mathrm{D}$ and $3 \mathrm{D}$ solutions for $\lambda$ has the order $1 / \Lambda^{2}$ for the cylinder, while the order is $1 / \Lambda$ for the most general long body.

\subsection{Analytical solutions for a finite cylinder}

\subsubsection{A complete closed-form solution}

The solution presented in the previous subsection is asymptotic for a thin body when the aspect ratio is large enough. However, there exist a few solutions for the finite cylinder to the problem involving constant Dirichlet boundary conditions. This will allow to precise for which lengths the $2 \mathrm{D}$ solution is convenient to represent the restriction of the $3 \mathrm{D}$ solution to the part of the midplane located near the boundary. These solutions involve either the "closed cylinder" comprising the lateral cylindrical boundary and plane circular boundaries at both tips of the cylinder $[34,35,36]$ or the hollow cylinder which comprises only the lateral cylinder $[37,38]$. The hollow cylinder corresponds therefore to a conductor whose orthogonal cross section is a thin circular layer.

The analytical solutions produce the distribution of surface charge density induced by a constant given potential of the conductor, which allows to compute the capacitance $Q / u$ involving the total charge on the conductor induced by a given surface potential $u$ and also the distribution of the potential in all the surrounding space. It was shown in [34] that the capacitance of the closed

cylinder is the same as the capacitance of the hollow cylinder as soon as the 
ratio $H / R$ is greater than 2 . Therefore, in the following, the results will be given only for the hollow cylinder.

The solution for the hollow cylinder which is used in the following was provided by Verolino [37]. The solution is completely defined by the surface density of charge at the surface of the cylinder. This density is defined on a circle at height $z$ by:

$$
\sigma(z)=\frac{2 u}{\sqrt{\alpha^{2}-z^{2}}} \sum_{p=1}^{\infty}(-1)^{p-1} C_{p} T_{2 p-2}(z / \alpha)
$$

where $T_{2 p-2}$ are Chebyshev polynomials and $u$ is the given constant potential.

The series coefficients $C_{p}$ are solutions of a linear system of equations $D_{n p} C_{p}=$ $\delta_{n 1}$ characterized by matrix coefficients $D_{n p}$ which are obtained by integrals of products of Bessel functions. Formula (32) shows that this solution is again singular at the tips of the cylinder.

It has been noticed during the computations that the expressions of the matrix coefficients $D_{n p}$ converge poorly and are unstable for large aspect ratios. Practically, However, having constructed the matrix of coefficients $D_{n p}$, the number of coefficients $C_{p}$ to use for the convergence of the series is moderate, typically inferior to 10 .

\subsubsection{A simplified closed-form solution}

As explained previously, the convergence of the matrix terms allowing the computation of the series coefficients in (31) is very unstable for large aspect ratios of the cylinder. So, an approximate solution given by Scharstein [39] will be also used in the following. It provides the charge density distribution with the simple form:

$$
\sigma(z)=\frac{u}{R}\left[c_{0}+\frac{c_{1}}{\sqrt{1-x^{2}}}\right]
$$

where $x=\frac{z}{\alpha}$ and $c_{0}$ and $c_{1}$ are explicit functions for large values of the aspect ratio.

It is shown in [39] that this solution recovers the solution provided by [38] for large aspect ratios of the cylinder.

\section{Solution obtained by the Boundary Element Method in the ax- isymmetric case}

As explained previously, studying the Dirichlet problem with a constant potential is very important for practical reasons, leading to the capacitance of useful systems. As seen in section 3, the solution of $2 \mathrm{D}$ problems for large values of aspect ratios produces asymptotically the solution of the $3 \mathrm{D}$ problem in the midplane, as soon as the characteristic length is chosen correctly. In order to evaluate the difference between $2 \mathrm{D}$ and $3 \mathrm{D}$ solutions in the intermediate range of aspect ratios, the problem of a long cylinder with a circular crosssection is solved in this section by using the Boundary Element Method. For 
any boundary conditions, this problem is fully 3D. A simplified version of the problem is the axisymmetric case where the boundary conditions on the cylinder do not depend of the azimuth. In this case, the problem can be solved as a $2 \mathrm{D}$ problem in a plane containing its axis. For this axisymmetric problem, the boundary conditions are fixed on the straight line (corresponding to $r=R$ ) characterizing the bounding cylinder. The Green's function to introduce into this $2 \mathrm{D}$ problem is the integral related to a circular line source located along a circle of radius $R$ located at the height $\zeta$ which produces the field at height $z$ given by:

$$
v(z, \zeta)=\frac{K\left(4 / p^{2}\right)}{\pi \cdot R \cdot p}
$$

where $p$ is related to the relative height $z-\zeta$ by:

$$
p=\frac{\sqrt{(z-\zeta)^{2}+4 \cdot R^{2}}}{R}
$$

and where $K$ is the complete elliptic integral of first kind.

The special function $K$ presents itself in the literature under two forms. The form corresponding to this formula is the one defined in formula 17.3.1, p.590 of[40], which is the same as the one used in Matlab software, but different from the notation used in Maple software.

Similarly, the integral over a circle of the normal gradient of the 3D Green's function is given by:

$$
w=\frac{1}{2 \pi \cdot R^{2} p}\left[K\left(\frac{4}{p^{2}}\right)-E\left(\frac{4}{p^{2}}\right)\right]
$$

where $E$ is the complete elliptic integral of second kind (formula 17.3.2, p.590 of [40]).

The integral equation for the potential $u$ becomes:

$$
u(z)+\int_{-a / 2}^{a / 2}[u(\zeta)-u(z)] w \cdot d \zeta=\int_{-a / 2}^{a / 2} v \cdot \frac{\partial u}{\partial \mathbf{n}} d \zeta
$$

The left integral is regular and $v$ has a weak logarithmic singularity. So, from a numerical point of view, this integral equation can be solved by the classical ways of Boundary Element Method for plane problems, using a discretization of the segment located between $z=-a / 2$ and $z=a / 2$ representing the section of the cylinder by a plane containing its axis. Three kinds of solutions were used in the following computations: constant elements, linear and quadratic elements. The results are identical for these three kinds of approximations.

\section{Numerical results}

\subsection{Dirichlet problem}

The different solutions which were presented in the previous sections are now used in order to be compared through different geometrical configurations, 
i.e. different values of the aspect ratio which is the ratio $a / R$ between height and radius of the cylindrical domain. As the $3 \mathrm{D}$ solution is assumed to produce results in the midplane which are comparable to the $2 \mathrm{D}$ solution, the values of impedance $q / u$, with $q=\frac{\partial u}{\partial \mathbf{n}}(z=0)$ are reported in Fig.5 as a function of the aspect ratio for the different types of solutions described previously. In this case, the potential at the cylinder is constant over its surface and the distribution of sources is computed along the cylinder.

From this figure, the full 3D solution of Verolino (1995) compares very well with the BEM solution for lower values of aspect ratio. For aspect ratios higher than 50, the solution of Scharstein (2007) and of Jackson (2000) become also satisfying, while for the highest values of aspect ratios, the full 3D solution departs from the others, due to numerical instabilities when computing the coefficients $C_{p}$ of formula (32), as mentioned previously in subsection 4.2. The $2 \mathrm{D}$ solution computed with a characteristic length equal to the length of the cylinder is then compared with these solutions and it can be seen that the $2 \mathrm{D}$ solution recovers the $3 \mathrm{D}$ solutions for aspect ratios higher than 100 , with a slight overevaluation by a few percent. The $3 \mathrm{D}$ solution can be compared with the $2 \mathrm{D}$ solution near the midplane, but departs obviously from the 3D solution due to end effect. This end effect is recovered by the 3D BEM solution (obviously with a finite end effect!), as it can be seen in Fig. 6 where the value of $q$ is reported as a function of the position along the cylinder. In addition, it can be seen that the value of $q$ is nearly constant in the region around the midplane $-25<z<25$ (approximately half the cylinder length) and recovers the $2 D$ value.

A last interesting result is the radial variation of the solution. Indeed, this radial variation in the midplane is drawn in Fig.7 for both 2D and 3D solutions, this result being obtained for an aspect ration $a / R=100$. It can be seen that both solutions are comparable in the vicinity of the cylinder (upto $r=10$ ), but that both solutions separate next, the $2 \mathrm{D}$ solution becoming obviously null for $r=a$, while the $3 \mathrm{D}$ solution tends smoothly to zero for high radii.

\subsection{Neumann problem}

In this case, all points at the surface of the cylinder have a constant radial gradient $q$ and the potential is computed along the cylinder. As the BEM solution was validated previously for other boundary conditions, this solution is used again with Neumann boundary condition for comparison with 2D solution in Fig. 8 where the impedance in the midplane is reported again as a function of the aspect ratio. It can be seen that the $2 \mathrm{D}$ solution produces values of impedance which are nearly the same as for 3D solution even with moderate aspect ratios as soon as $H / a \geq 5$. The variation of the potential along the length of the cylinder is reported in Fig.9 where it can be seen that there is no more singularity at the tip of the cylinder and that the solution near the midplane is correctly approximated by the $2 \mathrm{D}$ solution. These results are in complete agreement with the asymptotic solution obtained in section 3 by the slender body theory. 


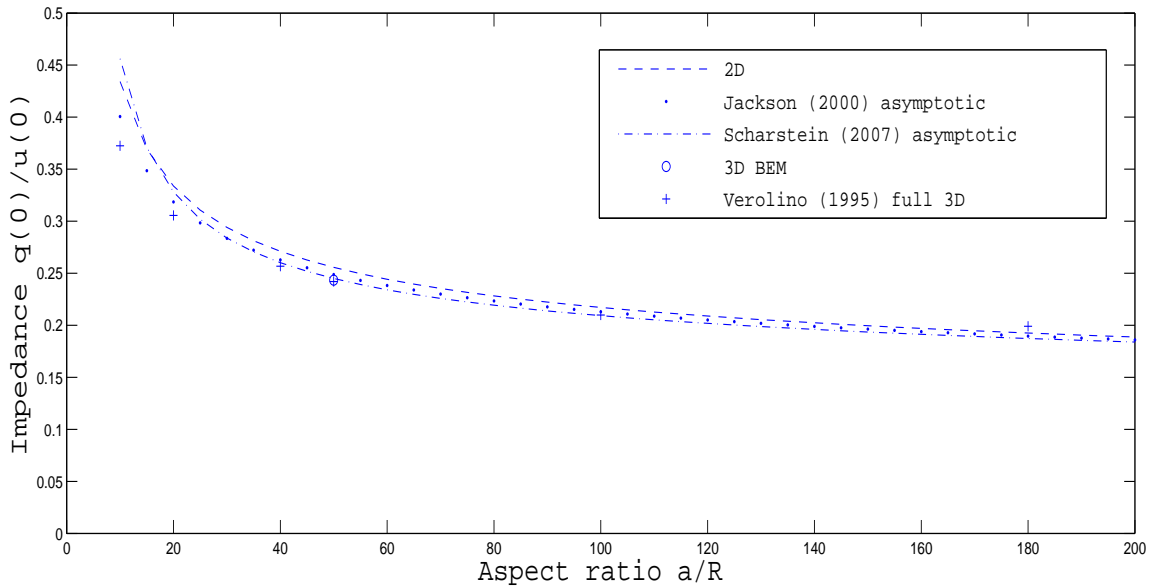

Figure 5: Dirichlet boundary condition: comparison of impedances coming in the midplane from fully analytical, asymptotic and numerical solutions.

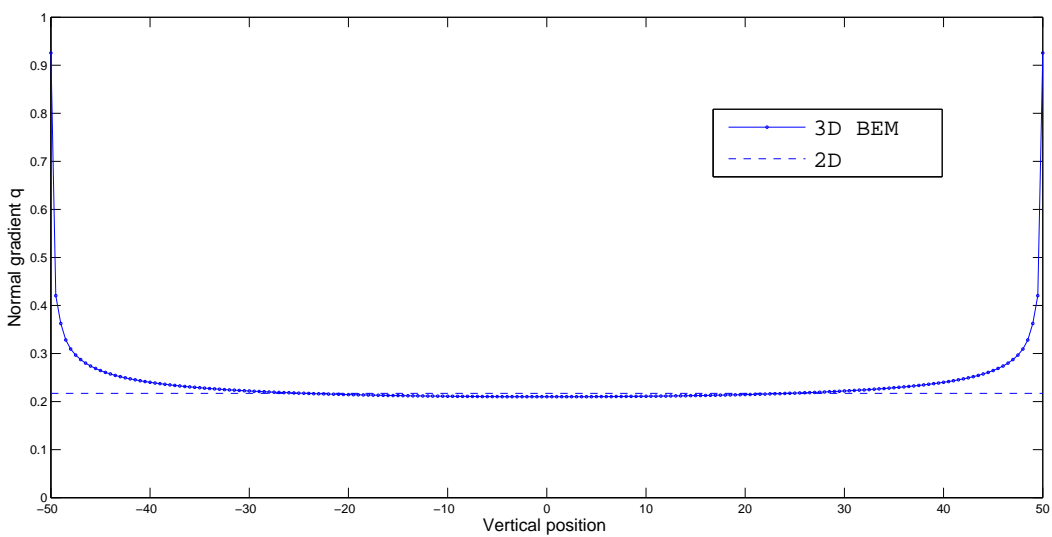

Figure 6: Dirichlet boundary condition: normal gradient along the cylinder as a function of the position for an aspect ratio $a / R=100$. 


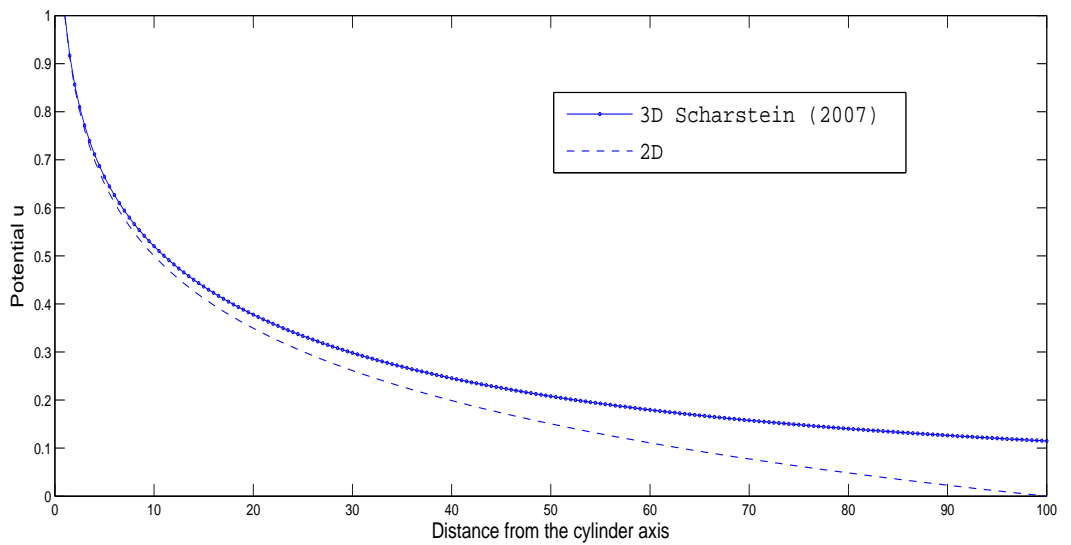

Figure 7: Dirichlet boundary condition: potential as a function of the radial position for 2D and $3 D$ solutions related to an aspect ratio $a / R=100$.

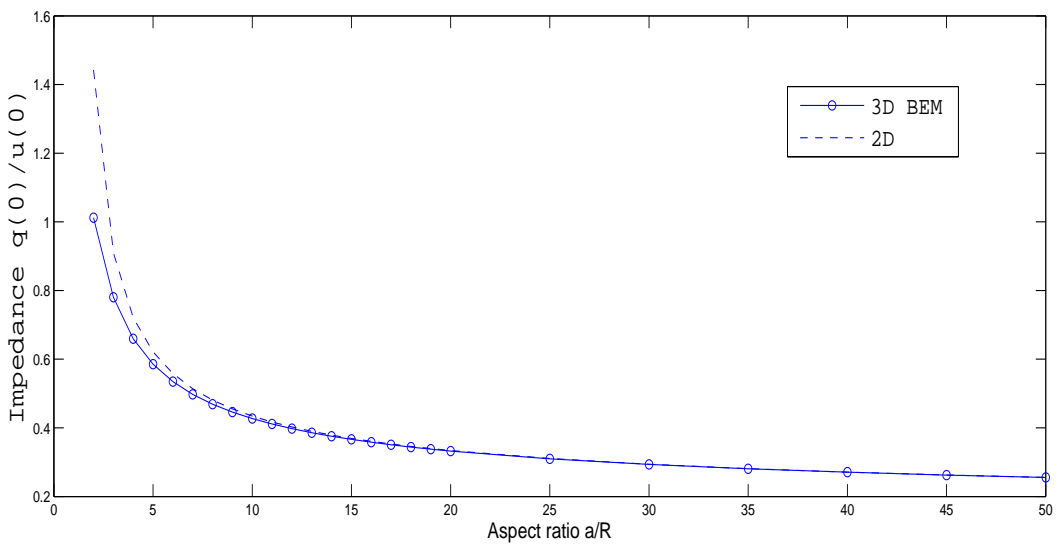

Figure 8: Neumann boundary condition: comparison of impedances in the midplane for $2 D$ and $3 D$ (BEM) solutions. 


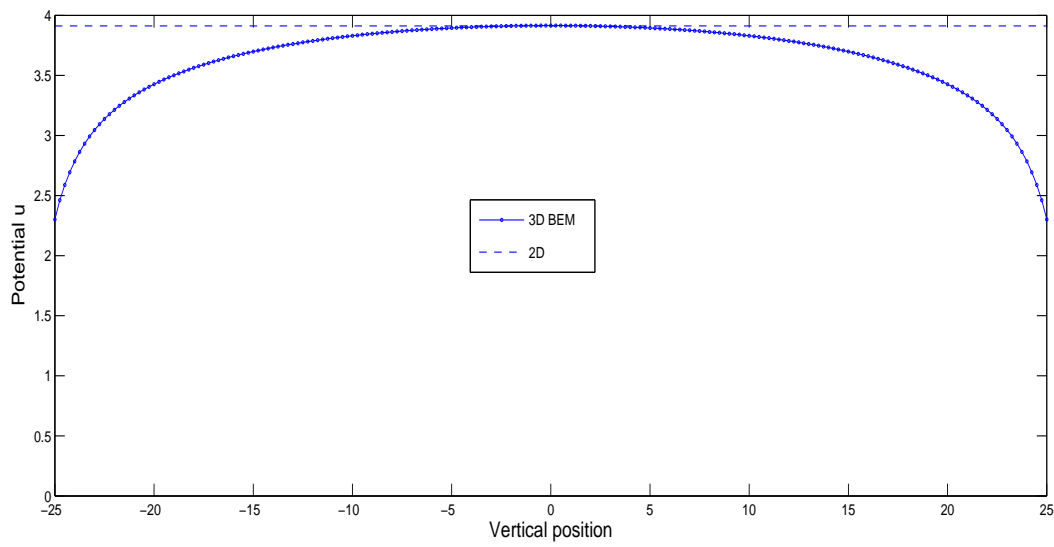

Figure 9: Neumann boundary condition: potential along the cylinder as a function of the position for an aspect ratio $a / R=50$. 


\section{Conclusion}

In many situations, the boundary element method is used as a complement to the finite element method for replacing an infinite part of the domain. An "impedance matrix" can be built from the boundary element formulation. It was shown in this paper that the use of the classical singular solution for Laplace equation combined with the boundary element formulation can lead for plane problems to "impedance matrices" which are not positive definite or which have very large eigenvalues. This leads to numerical problems when the combined FEM-BEM problem is solved. This problem is close to the classical question of the non-uniqueness of the Dirichlet's boundary value problem for Laplace's equation. Indeed, it is known that the Green's function for plane problems is defined upto a constant term and that this constant must be large enough to ensure uniqueness. Unfortunately, if for the interior problem with Dirichlet or mixed boundary conditions, the solution does not depend on the added constant, it is no more the case for the outer problem: the solution to these problems depend explicitly of the added constant. So, it is crucial to provide a clear procedure for defining such a constant, or in an equivalent way, the characteristic length to introduce into the Green's function.

In order to define the characteristic length to introduce into the Green's function, it is convenient from a physical point of view to consider a2D problem as the limit of a 3D problem on a very long, but finite, cylindrical domain along which boundary conditions do not depend on the position variable along the direction of the length of the cylinder. The $2 \mathrm{D}$ solution is then the limit of the $3 \mathrm{D}$ solution for large values of the length of the cylinder. Studying this problem by the "slender body theory" has produced the value of the characteristic length to introduce into the Green's function, which is exactly the length of the cylinder.

This result has been verified by using solutions for a large cylinder submitted to Dirichlet or Neumann boundary conditions. All results confirm the value of the characteristic length and show that the $2 \mathrm{D}$ solution produces an estimation of the 3D solution near the cylinder in the vicinity of the midplane. This result

confirms also that the 2D BEM solution can be used even if the resultant of the flux on the interior boundary is not null, as shown in [11]. Finally, the 2D Green's function is completely defined, the value of the characteristic length being completely defined by the associated 3D problem, leading to a "physical Green's function". From a practical point of view, the field produced by the "physical Green's function" can also be seen as the one produced by a line source whose length is the height of the cylinder along the direction orthogonal to the computation plane.

This paper has been devoted to Laplace equation, but an extension to problems related to elasticity equations will be the subject of a future work. 


\section{Appendix A. Appendix: Derivation of the impedance matrix from classical integral equation}

Let us consider the solution $u$ of the exterior problem of Laplace equation on a plane domain $D$ having a boundary $\partial D$ for Dirichlet boundary conditions.

$u$ is therefore the solution within $D$ of:

$$
\Delta u=0,
$$

with the boundary conditions $u=u_{0}$ on $\partial D$.

The classical direct formulation of the boundary element method rests on the formulation of a boundary integral equation on $\partial D$. Different expressions of that integral equation exist, either involving "free terms" or using regularized expressions. As soon as a higher order interpolation of the solution on the boundary is used, the regularized formulation is the most efficient.

Let us therefore start from the regularized integral equation for the exterior problem obtained in [4], which rests on the regularization method introduced by [41]:

$$
u(\mathbf{x})+\int_{\partial D}\{[u(\mathbf{y})-u(\mathbf{x})] H(\mathbf{x}, \mathbf{y})-q(\mathbf{y}) G(\mathbf{x}, \mathbf{y})\} \mathrm{d} S_{y}=0
$$

where $G$ is the Green's function for Laplace equation and $H$ the normal derivative computed from $G$. Points $\mathbf{x}$ and $\mathbf{y}$ are on the contour, while $u$ and $q$ are the boundary values of the potential and of its normal gradient.

It is shown in [4] that the integral on the term containing $H$ converges simply and that it is not necessary to use a convergence of the "Cauchy" type as for other formulations.

The potential within $D$ is given from the values of the potential and of the flux on the boundary by the representation:

$$
u(\mathbf{x})=\int_{\partial D}\{q(\mathbf{y}) G(\mathbf{x}, \mathbf{y})-u(\mathbf{y}) H(\mathbf{x}, \mathbf{y})\} \mathrm{d} S_{y}
$$

The discretized boundary element formulation of that equation, using for example a linear interpolation on all elements is:

$$
[H][u]=[G][q]
$$

where $[u]$ and $[q]$ contain the nodal displacements and the nodal values of the normal flux while $[H]$ and $[G]$ are built from the interpolation functions, the geometry of the elements and from the Green's function (and its derivatives).

One possibility to perform the coupling between the formulation of the exterior problem and a finite element solution within the interior domain is to obtain the "impedance matrix" which allows to compute the "concentrated nodal flux" contained in matrix $[F]$ from the nodal values of the approximate potential.

This impedance matrix is given by [26]:

$$
\left[K_{1}\right]=[Q][G]^{-1}[H]
$$


where $[Q]$ is built by summing the contributions of each element $e_{m}$ defined by:

$$
Q_{i j}=\int_{e_{m}} N_{i}(\xi) N_{j}(\xi) J(\xi) \mathrm{d} \xi
$$

\section{References}

[1] V. D. Kupradze, Potential methods in the theory of elasticity, Israel Program for Scientific Translation, Jerusalem, 1965.

[2] M. Maiti, S. S. Palit, Somigliana's method applied to plane problems of elastic half spaces, Journal of Elasticity 6 (1976) 429-439.

[3] J. O. Watson, Advanced implementation of the boundary element method for two- and three-dimensional elastostatics. in Developments in boundary element methods 1, edited by Banerjee and Butterfield, pp.31-63, Applied Science, London, 1987.

[4] M. Bonnet, Boundary Integral Equations Methods for Solids and Fluids, Wiley, N.Y., 1999.

[5] C. Constanda, On non-unique solutions of weakly singular integral equations in plane elasticity, Quarterly Journal of Mechanics and Applied Mathematics 47 (2) (1994) 261-268.

[6] C. Constanda, Direct and indirect boundary integral equation methods, Monographs and surveys in pure and applied mathematics N107, Chapman and Hall/CRC, Boca Raton, 1999.

[7] P. Schiavone, C. Q. Ru, On the exterior mixed problem in plane elasticity, Mathematics and Mechanics of Solids 1 (1996) 335-342.

[8] G. Hsiao, R. C. Maccamy, Solution of boundary value problems by integral equations of the first kind, SIAM Review 15 (4) (1973) 687-705.

[9] M. H. Aliabadi, The boundary element method: Applications in solids and structures, Wiley, N.Y., 2002.

[10] Y. Z. Chen, X. Y. Lin, Regularity condition and numerical examination for degenerate scale problem of BIE for exterior problem of plane elasticity, Engineering Analysis with Boundary Elements 32 (2008) 811-823.

[11] A. Corfdir, G. Bonnet, Validity conditions of the direct boundary integral equation for exterior problems of plane elasticity, Comptes Rendus Mecanique 335 (4) (2007) 219-224.

[12] M. A. Jaswon, Integral equation methods in potential theory, I, Proceedings of the Royal Society of London A275 (1963) 23-32. 
[13] P. K. Banerjee, R. Butterfield, Boundary element methods in engineering science, McGraw-Hill, N.Y., 1981.

[14] Y. Z. Chen, X. Y. Lin, Z. X. Wang, Numerical solution for degenerate scale problem for exterior multiply connected region, Engineering Analysis with Boundary Elements 33 (2009) 1316-1321.

[15] G. Hsiao, On the stability of integral equations of the first kind with logarithmic kernels, SIAM Review 94 (2) (1986) 179-192.

[16] J. T. Chen, S. R. Kuo, J. H. Lin, Analytical study and numerical experiments for degenerate scale problems in the boundary element method for two-dimensional elasticity, International Journal for Numerical Methods in Engineering 54 (12) (2002) 1669-1681.

[17] J. T. Chen, W. C. Chen, S. R. Lin, I. L. Chen, Rigid body mode and spurious mode in the dual boundary element formulation for the Laplace problems, Computers and Structures 81 (13) (2003) 1395-1404.

[18] J. T. Chen, S. R. Lin, K. H. Chen, Degenerate scale problem when solving Laplace's equation by BEM and its treatment, International Journal for Numerical Methods in Engineering 62 (2) (2005) 233-261.

[19] R. Vodicka, V. Mantic, On invertibility of elastic single-layer potential operator, Journal of Elasticity 74 (2) (2004) 147-173.

[20] R. Vodicka, V. Mantic, On solvability of a boundary integral equation of the first kind for Dirichlet boundary value problems in plane elasticity, Computational Mechanics 41 (6) (2008) 817-826.

[21] S. Christiansen, Integral equations without a unique solution can be made useful for solving some plane harmonic problems, Journal of the Institute of Mathematics and Its Applications 16 (1975) 143-159.

[22] S. Christiansen, Derivation and analytical investigation of three direct boundary integral equations for the fundamental biharmonic problem., Journal of Computational and Applied Mathematics 91 (2) (1998) 231247.

[23] J. Chen, C. Wu, K. Chen, Y. Lee, Degenerate scale for the analysis of circular thin plate using the boundary integral equation method and boundary element methods, Computational Mechanics 38 (1) (2006) 33-49.

[24] Y. Z. Chen, X. Y. Lin, Degenerate scale problem for the Laplace equation in the multiply connected region with outer elliptic boundary, Acta Mechanica 225 (2010) 215-233.

[25] W. Dijkstra, R. M. M. Mattheij, A relation between the logarithmic capacity and the condition number of the BEM matrices, Communications in Numerical Methods in Engineering 23 (2007) 665-680. 
[26] C. A. Brebbia, P. Georgiou, Combination of boundary and finite elements in elastostatics, Applied Mathematical Modelling 3 (1979) 212-220.

[27] E. Tuck, Y. Stokes, On thin or slender bodies, Australian and New Zealand Industrial and Applied Mathematics Journal 53 (2012) 190-212.

[28] E. Chadwick, A. Hatam, Slender body expansions in potential theory along a finite straight line, Zeitschrift fur angewandte Mathematik und Physik 61 (2010) 493-508.

[29] G. I. Sivashinsky, The problem of a slender die, Journal of Elasticity 5 (1) (1975) 161-166.

[30] E. Tuck, C. Mei, Contact of one or more slender bodies with an elastic half space, International Journal of Solids and Structures 19 (1) (1983) 1-23.

[31] Y. Yan, I. H. Sloan, On integral equations of the first kind with logarithmic kernels, Journal of Integral Equations and Applications 1 (4) (1988) 549579 .

[32] J. Maxwell, On the electric capacitance of a long narrow cylinder and a disk of sensible thikness, Proceedings of the London mathematical society 9 (1878) 94-101.

[33] J. Jackson, Charge density on a thin straight wire, revisited, American Journal of Physics 68 (9) (2000) 789-799.

[34] S. Falco, G. Panariello, F. Schettino, L. Verolino, Capacitance of a finite cylinder, Electrical engineering 85 (2003) 177-182.

[35] W. Smythe, Charged right circular cylinder, Journal of Applied Physics 27 (8) (1956) 917-920.

[36] W. Smythe, Charged right circular cylinder, Journal of Applied Physics 33 (10) (1962) 2966-2967.

[37] L. Verolino, Capacitance of a hollow cylinder, Electrical engineering 78 (1995) 201-207.

[38] L. Vainshteyn, Static boundary problems for a hollow cylinder of finite length. Part II, Technical physics-Soviet physics 7 (1963) 855-860.

[39] R. Scharstein, Capacitance of a tube, Journal of Electrostatics 65 (2007) 21-29.

[40] M. Abramowitz, I. Stegun, Handbook of Mathematical Functions with Formulas, Graphs, and Mathematical Tables, Dover, N.Y., 1972.

[41] F. J. Rizzo, D. J. Shippy, M. Rezayat, A boundary integral equation method for radiation and scattering of elastic waves in three dimensions, International Journal for Numerical Methods in Engineering 21 (1985) 115-129. 